
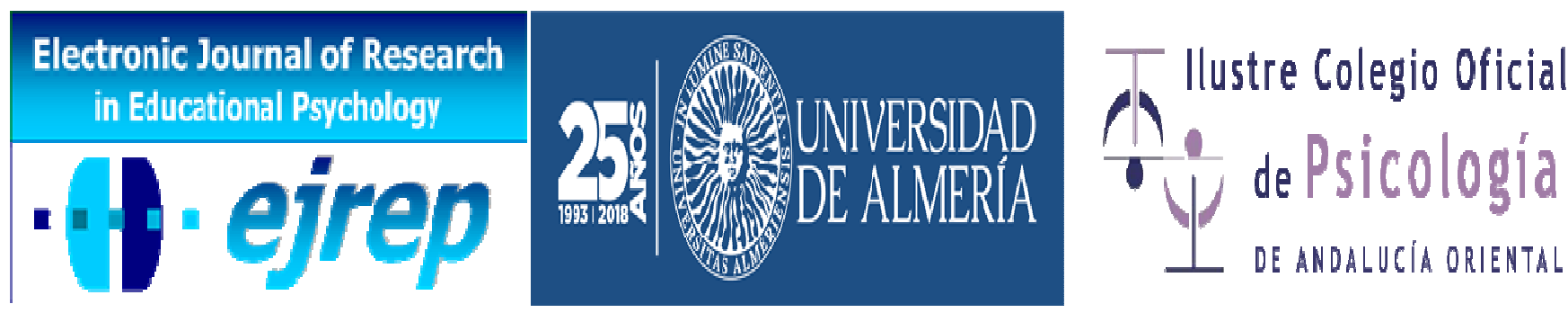

\title{
Revisores 2017
}


Adriana Gladys, Faviery. UTN (Argentina)

Alemany, Inmaculada. Universidad de Granada (España)

Alonso Tapia, Jesús. Universidad Autónoma de Madrid (España)

Al-Qaryouti, Ibrahim. Sultan Qaboos University (Oman)

Alvarez, Blas. Universidad de Lima (Perú)

Aparisi, David. Universidad de Alicante (Spain)

Ato Lozano, Ester. Universidad de Murcia (España)

Balcikanli, Cem. Gazi Universitesi (Turquía)

Boato, Yanina. Universidad de Río Cuato (Argentina)

Burger-Veltmeijer, Agnes E.J. University of Groningen (The Netherlands)

Benítez, J. Luis. Universidad de Granada (España)

Castelló, Montserrat. Universitat Ramón Lull (España)

Cejudo, Javier. Facultad Educacion, Ciudad Real (España)

Cerezo Rucillo, Teresa. Universidad de Jaén (España)

Chávez Soto, Blanca Ivet (Mexico)

Clariana, Mercè. Universidad Autónoma de Barcelona (España)

Chavez Soto, Blanca. Universidad Autónoma de México (México)

Dejonckheere, Peter. Katho PHO (Belgium)

Dereli-İman, Esra. Eskişehir-Turkey (Turquía)

Doménech Betoret, Fernando. Univeridad Jaume I (España)

Faber, Günter. Leibniz University Hannover (Germany)

Félix Mateo, Vicente. Hospital Clínico de Valencia (España)

Filella Guiu, Gemma.Universidad de Lleida (España)

Fleming, Manuela. Universidad de Porto (Portugal)

Furlam, Luis Alberto. Univeridad Nacional de Córdoba (Argentina)

Gadelrab, Hesham F. Umm Alqra University (Arabia Saudi)

González Salinas, Carmen. Universidad de Murcia (España)

Gutiérrez, Rosaura. UGR (República Dominicana)

Jiménez Rodríguez, Virginia. Universidad Complutense de Madrid (España)

Justicia, Fernando. Universidad de Granada (España)

Hernández, Carmen. Univeridad de la Laguna (España)

Hilpert, Jonathan C. Georgia Suthem University (USA)

Korzeniowski, Celina. CONICET (Argentina)

Kosmala-Anderson, Joanna Coventry University (UK)

León del Barco, Benito. UNEX (España) 
Marugán, Montserrat.Universidad de Valladolid (Spain)

Martínez Carpio, Héctor. Universidad Católica de Santa María, Arequipa (Perú)

Mateos, Mar. Universidad Autónoma de Madrid (España)

Melagakaki, Olga. University of Picar (France)

Méndez, Marta. Universidad de Oviedo (España)

Nyroos, Mykaela. Umea University (Sweden)

Omyeizugbo, Euckay U. University of Nigeria (Nigeria)

Osborne, Jason. Nord Carolina University (USA)

Papantoniou, Georgia. University of Ioannina (Greece)

Paredes Gómez, Daniel. Centro Ocupacional Aprosuba-4. Extremadura (España)

Papantoniou, Georgia. University of Ioannina (Greece)

Paulino, Paula. University of Lisbon (Portugal)

Pena Garrido, Mario. UNED (España)

Peralta, Felicidad. Universidad de Navarra (España)

Pérez-Escoda, Núria. Universitat de Barcelona (España)

Pozo, Carmen. Universida de Almería (España)

Ribeiro Viseu, João Nuno. Research Centre for Spatial and Organizational Dynamics (Portugal)

Rosser Limiñana, Ana María. Universidad de Alicante (España)

Shake, Matthew C. Western Kentuky University (USA)

Pelagajar, María del Carmen. Universidad de Murcia (España)

Sander, Paul. Arden University (UK)

Shoor, Cornelia. University of Bamber (Germany)

Sommer, Lutz. Albstadt Sigmairngen University (Germany)

Verónica Paoloni, Paola. Universidad de Río Cuato (Argentina)

Yoncalik, Oguzhan. Egitim Fakültesi (Turquía) 
META-DATOS 2017

Recibidos: 104 articulos

Rechazo inicial: 50 articulos

Rechazo Final: 24 articulos

Aceptados: 30 articulos

Tasa de aceptación: $28.84 \%$

Tasa de Rechazo: $71.15 \%$ 